\title{
Insulin secretion and impaired glucose tolerance. Reply to Jenkins AB, Campbell LV [letter]
}

\author{
A. Mari • E. Ferrannini
}

Received: 18 May 2010 / Accepted: 21 May 2010 / Published online: 11 June 2010

(C) Springer-Verlag 2010

Keywords Beta cell compensation - Beta cell function . Disposition index · Glucose tolerance $\cdot$ Insulin sensitivity . Hyperglycaemia · Mathematical modelling · Pre-diabetes . Type 2 diabetes

\section{Abbreviation \\ NGT Normal glucose tolerance}

To the Editor: Drs Jenkins and Campbell recall a basic equation of glucose homeostasis (equation 1 in Jenkins and Campbell [1]) that is indeed useful for understanding the interrelationships between beta cell function, insulin sensitivity and glucose tolerance. This equation illustrates well their critique of the disposition index, on which we have also expressed some reservations [2, 3].

However, we think that their analysis may miss a central point, i.e. the nature and physiological meaning of these relationships. They say that the disposition index 'theory was originally developed to explain the hyperbolic relationship between insulin secretion and insulin sensitivity, which within our model is an intrinsic property resulting from the underlying physiology requiring no additional

\footnotetext{
A. Mari $(\bowtie)$

ISIB-CNR,

Corso Stati Uniti 4,

35127 Padova, Italy

e-mail: andrea.mari@isib.cnr.it

E. Ferrannini

Department of Internal Medicine and C.N.R.

Institute of Clinical Physiology,

University of Pisa School of Medicine,

Pisa, Italy
}

mechanistic explanation' [1]. It seems to us that this view ignores the cause-effect relationship. It is the existence of a physiological inverse relationship between beta cell function $(\beta)$ and insulin sensitivity $\left(S_{\mathrm{i}}\right)$ that makes glucose $(G)$ relatively constant, as $G$ is determined by $\beta$ and $S_{\mathrm{i}}$ (assuming that glucose appearance, $R_{\text {in }}$ in Eq. 1 of Jenkins and Campbell [1], is of minor importance). If, conversely, $\beta$ and $S_{\mathrm{i}}$ were unrelated, $G$ would not be equally constant, but obviously the lack of the $\beta-S_{\mathrm{i}}$ relationship would be the primary fact and not a consequence of a relationship masked by large variations in $G$.

These arguments apply to Fig. 1c of our article [4]: we think that the lack of a significant relationship between $\ln (\beta)$ and $\ln \left(S_{\mathrm{i}}\right)$, when the glucose range $(\ln (G))$ is not too narrow, is not due to the existence of an underlying $\beta-S_{\mathrm{i}}$ relationship masked by $\ln (G)$ variations. On the contrary, it is the lack of relationship between $\ln (\beta)$ and $\ln \left(S_{\mathrm{i}}\right)$ that makes $\ln (G)$ so strongly dependent on $\ln (\beta)$.

In other words, equation 1 of Jenkins and Campbell [1] is a constraint that can be read: 'if $S_{\mathrm{i}}$ is inversely related to $\beta$ then one can expect that glucose is relatively constant; otherwise, glucose will vary in relation to $\beta$ and $S_{\mathrm{i}}$ '. Thus, it is the existence or non-existence of a physiological relationship between insulin sensitivity and beta cell function that will determine the glucose levels.

The correlation between insulin sensitivity and beta cell function is subject to direct testing, as always, keeping in mind the statistical characteristics of the indices used. In our study [4], the expected estimation errors associated with the various beta cell function parameters are not very different [5], yet in 1,123 individuals with normal glucose tolerance (NGT) glucose sensitivity was not correlated with insulin sensitivity while several other parameters were (including, as expected, the model surrogate of the acute 
insulin response, rate sensitivity, which has the lowest reproducibility [5]; Spearman rho $-0.15, p<0.0001$ in NGT, data not reported in Mari et al. [4]). Therefore, based on the data from our study a relationship between glucose sensitivity and insulin sensitivity cannot be established, while it exists for other parameters (as in Fig. 1a of our article [4]).

In this discussion, it is useful to recall that the concept of a disposition index was prompted by the observation of an inverse relationship between $\beta$ and $S_{\mathrm{i}}$ in cross-sectional data from healthy NGT individuals (such as in our Fig. 1a) [3], without an experimental demonstration of a causal link (e.g. at the level of beta cells) between insulin sensitivity and beta cell function. In other words, beyond correlation there is no basis for assuming a $\beta-S_{\mathrm{i}}$ relationship. Therefore, there is no basis for hypothesising that such a correlation exists but is masked by factors such as glucose levels. One can always assert that our Fig. 1c hides a family of hyperbolas (or whatever functions) stacked together to cover the cloud of points, but this view stems from a paradigm based solely on the existence of a correlation that is contradicted by Fig. 1c.

Indeed, the pioneering work on the disposition index concept by Bergman and colleagues was based on an intervention protocol in the dog, in which diet-induced changes in insulin sensitivity were accompanied by reciprocal changes in some insulin secretion parameter [3]. One may think that this is a stronger approach; unfortunately, it does not overcome an objection analogous to that of the hidden hyperbolas. Nevertheless, in our own longitudinal studies of non-diabetic patients undergoing surgical weight loss $[6,7]$ we found that fasting and total insulin secretion decreased as insulin sensitivity increased (as expected), while beta cell glucose sensitivity was not affected. Thus, our follow-up studies are perfectly in line with the interpretation that adaptation is specific of only some insulin secretion parameters, as we discuss in Mari et al. [4].
We hope that future studies will shed light on the compensation mechanisms, perhaps at a cellular level, bypassing the difficulties inherent in cross-sectional correlation and follow-up studies. In the meantime, we prefer to stick to the simpler view that adaptation is specific of some insulin secretion parameters. The arguments on universal compensation masked by confounding factors seem to us as artificial as Ptolemy's epicycles must have appeared to Copernicus.

Duality of interest The authors declare that there is no duality of interest associated with this manuscript.

\section{References}

1. Jenkins AB, Campbell LV (2010) Insulin secretion and impaired glucose tolerance. Diabetologia. doi:10.1007/s00125-010-1801-1

2. Ferrannini E, Mari A (2004) Beta cell function and its relation to insulin action in humans: a critical appraisal. Diabetologia 47:943956

3. Mari A, Ahrén B, Pacini G (2005) Assessment of insulin secretion in relation to insulin resistance. Curr Opin Clin Nutr Metab Care 8:529-533

4. Mari A, Tura A, Natali A et al (2010) Impaired beta cell glucose sensitivity rather than inadequate compensation for insulin resistance is the dominant defect in glucose intolerance. Diabetologia 53:749-756

5. Utzschneider KM, Prigeon RL, Tong J et al (2007) Within-subject variability of measures of beta cell function derived from a $2 \mathrm{~h}$ OGTT: implications for research studies. Diabetologia 50:25162525

6. Ferrannini E, Camastra S, Gastaldelli A et al (2004) beta-cell function in obesity: effects of weight loss. Diabetes 53(Suppl 3): S26-S33

7. Mari A, Manco M, Guidone C et al (2006) Restoration of normal glucose tolerance in severely obese patients after bilio-pancreatic diversion: role of insulin sensitivity and beta cell function. Diabetologia 49:2136-2143 